\title{
AUTOMATED GENERATION OF QUESTIONS FOR BASIC ELECTRICAL ENGINEERING EDUCATION
}

\author{
Frano Skopljanac-Macina, Bruno Blaskovic \& Damir Pintar
}
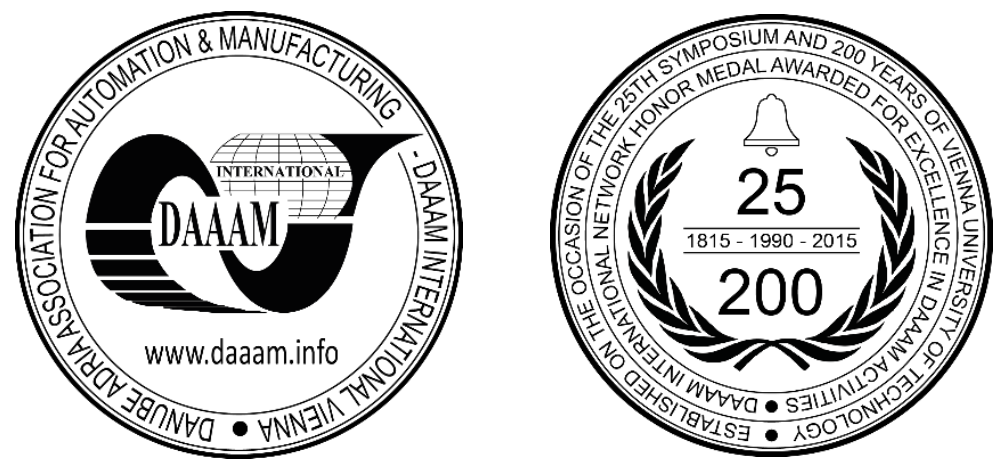

This Publication has to be referred as: Skopljanac-Macina, F[rano]; Blaskovic, B[runo] \& Pintar, D[amir] (2016). Automated Generation of Questions for Basic Electrical Engineering Education, Proceedings of the 27th DAAAM International Symposium, pp.0377-0385, B. Katalinic (Ed.), Published by DAAAM International, ISBN 978-3-90273408-2, ISSN 1726-9679, Vienna, Austria

DOI: $10.2507 / 27$ th.daaam.proceedings.056

\begin{abstract}
This paper deals with the problem of automated generation of questions for basic electrical engineering education in elearning systems. One of the main challenges in developing e-learning systems is to create an effective knowledge testing module. In most of e-learning systems teachers need to manually compose test questions, prepare the correct and wrong answers, and added feedback information. The process is time-consuming and prone to errors. We have focused on problems in basic electrical engineering education, specifically series - parallel DC circuits, and we propose a solution for automated generation of template based questions of various degrees of similarity and complexity. Our prototype Web application dynamically generates text of the question and its circuit diagram image, solves the problem, and as a feedback provides correct results and a step-by-step solution. The interface is bilingual, available in Croatian and English. We have used standard Web technologies, such as JavaScript, jQuery and SVG, together with MathJax for mathematical notation. We will recommend our students on the course Fundamentals of Electrical Engineering to use this Web Application for self-assessment and practice. In future work we plan to advance our Web application and to fully integrate it into our developing e-learning system.
\end{abstract}

Keywords: Automated question generation; automated feedback generation; e-learning systems; random circuit diagram generation.

\section{Introduction}

E-learning systems use computer technology and Internet in the field of education. E-learning is primarily used in formal education to support teaching and learning process. Today, e-learning systems are also used in corporate environment as a tool for training and testing current and future employees. E-learning is also used semi-formally for distance education in MOOCs (Massive online open courses), and informally in collaborative and life-long education, e.g. online (video) tutorials, social network groups and forums [1].

Today, e-learning systems are getting more complex, combining advantages of Intelligent Tutoring Systems (ITS) its individual approach to each student and student-tutor two way communication, with those of Adaptive Hypermedia Systems (AHS) - adaptable presentation of learning materials and choice of different learning paths. Such advanced elearning systems are known as Adaptive Web-based Educational Systems [2]. In universities they are usually based on 
the Moodle learning management system [3]. These systems facilitate learning process by providing students with individual adaptive learning environment, but also they can be efficiently used in training of teachers and administrative staff [4]. Online educational technologies can be used to create inter-university networks to connect, share knowledge and to remotely train specialists in different fields [5].

Nevertheless, teachers and instructors must invest a lot of their time and resources in creating and maintaining teaching and learning online materials, basic learning scenarios and learning paths. Finally, the process of devising test questions and preparing testing materials is very important, but usually delicate and time-consuming. For each new question instructors must also give the correct and the wrong answers, sometimes also draw a figure, and preferably add useful hints.

Recently, there is growing research interest in the field of question generation. It deals with the dynamic creation of questions for a specific knowledge domain. Some of the research is based on Natural language processing and Natural language generation, and the goal is to automatically create textual questions based on a given set of factual information. This information can be (semi-)structured (concept maps or OWL ontologies) or even unstructured (free text from articles or handbooks) [6].

There are also interesting research directions in question and problem generation for STEM field education (Science, Technology, Engineering and Mathematics). Usually, questions in these fields contain not only text, but also figures and/or formulae. Gulwani et al. in [7], [8], [9] and [10] researched how to dynamically generate tests and questions, as well as useful feedback information in the STEM field education. In [8] the authors propose a model for automatic generation of rich feedback information when a student submits wrong solution to a simple programming assignment on the course Introduction to programming in Python. However, teacher must first prepare an example of a correct implementation for each assignment, as well as typical coding errors. The system then tries to automatically correct student's wrong solution. If it succeeds, the system gives student instant feedback with short explanations together with the list of coding errors. Authors report that for $64 \%$ of wrong solutions the system was able to correct them, and provide useful feedback to students. In [9] the authors present an interesting synthesis method of geometric proofs based on a given figure (110 figures scanned from Indian and American high school mathematics handbooks) and a defined set of axioms. In [10] the authors propose a method for automatic generation of new variants of algebra problems, based on original problems from mathematical handbooks. Zitko et al. in [11] presented their ITS system for automatic test generation for evaluating knowledge contained in an OWL ontology. Test provides dynamically generated questions based on a predefined set of generic question templates.

In this paper we will focus on calculation questions in the field of basic electrical engineering. These questions contain text and figures with electrical circuit schema. We asked ourselves is there an effective way to easily generate dynamic questions of this type? That would benefit the assessment process - it would ensure that the most students get different questions of similar complexity.

The rest of the paper is structured as follows: Section 2 contains information about our e-learning system, and the motivation for this research; in Section 3, first we set prerequisites and outcomes of proposed automated question generation method, and then describe its implementation; in Section 4 we discuss the main results of presented research; and Section 5 contains conclusion and an overview of future work.

\section{Background}

We have developed our own e-learning system WebOE, which is primarily used on the freshman year course (over 650 students) Fundamentals of Electrical Engineering at University of Zagreb (Croatia), Faculty of Electrical Engineering and Computing [12]. The professors can use it to check on students' progress during the semester, upload teaching materials, give homework and quizzes, prepare written and oral exams, and analyze their results. And vice versa, students use it keep track of their success and obligations during the course, access various teaching/learning materials, solve homework and online quizzes, and check timetables for laboratory assignments. The WebOE system questions database currently contains 2987 questions as it is shown in Table 1.

\begin{tabular}{|c|c|c|}
\hline Question type & Static/dynamic content & Number of questions \\
\hline multiple choice ( 1 correct answer) & static & 2143 \\
\hline multiple choice ( 2 or more correct answers) & static & 166 \\
\hline fill in & static & 32 \\
\hline yes/no & static & 33 \\
\hline general questions (oral and written exam) & static & 171 \\
\hline homework (calculation) & dynamic & 264 \\
\hline $\begin{array}{c}\text { variable multiple choice questions } \\
\text { (1 correct answer, multiple variations of each question) }\end{array}$ & dynamic & 178 \\
\hline \multicolumn{2}{|l|}{ Total } & 2987 \\
\hline
\end{tabular}

Table 1. Question database in WebOE e-learning system 
From the Table 1 we can see that our question database is very rich, but nevertheless it is obvious that the majority of questions are static (2545) - question text, list of answers and an image (if present) are fixed. But, even with these static questions we can generate tests dynamically, by randomly selecting questions or with permutations of defined question lists. Also, the answer list in multiple choice questions can be permuted. Homework calculation questions are somewhat dynamic, because every student gets a unique combination of numeric inputs and different numeric solutions. Each homework calculation question must have its solution program for automatic checking and grading students' answers. But, the question text and the image are fixed so it is possible that more students get basically the same questions. Variable multiple choice questions are very dynamic, and we primarily use them for Laboratory quizzes. Each of those questions usually has 4 variations: question text can be substantially different in every variation, and different figures and answer lists can be used for each variation. Still, all of these questions have been prepared, checked and entered into the database manually.

\begin{tabular}{|c|c|c|c|}
\hline Year & Type of questions & Number of students & Success rate \\
\hline 2007 & static multiple choice & 794 & $49.11 \%$ \\
\hline 2008 & static multiple choice & 718 & $76.27 \%$ \\
\hline 2009 & static multiple choice & 832 & $75.94 \%$ \\
\hline 2010 & static multiple choice & 764 & $75.86 \%$ \\
\hline 2011 & static multiple choice & 821 & $81.53 \%$ \\
\hline 2012 & mixed (static \& variable multiple choice) & 771 & $71.21 \%$ \\
\hline 2013 & variable multiple choice & 622 & $56.24 \%$ \\
\hline 2014 & variable multiple choice & 563 & $53.20 \%$ \\
\hline 2015 & variable multiple choice & 667 & $58.40 \%$ \\
\hline 2016 & variable multiple choice & 656 & $56.96 \%$ \\
\hline
\end{tabular}

Table 2. Laboratory quizzes success rate (third laboratory test from 2007 to 2016)

As expected, the success rate of unchanged static questions rises with every new generation of students because of their collaborative work in gathering old questions and remembering correct answers (e.g. compare 2008 to 2007 success rate in Table 2). The success rate of old homework calculation questions also slowly rises every new academic year. Each year a number of old static questions and homework assignments have to be deactivated and manually replaced with their new variations. The variable questions have proved to be more resilient - since 2013 their success rate is more constant, oscillating below $60 \%$ success rate as can be seen in Table 2. That is much more representative than before 2012 when we used only static questions in laboratory quizzes (success rate was over $75 \%$ ). Different variations, sometimes very subtle, successfully confuse those students who did not bother to learn, but instead tried to memorize correct answers.

Rich instant feedback can be very valuable to students, and can motivate them to continue learning and practicing online, especially in distance learning [13]. Our system offers basic feedback (is the answer correct or not, and some statistical data). Additionally, some questions have useful rich explanations, but their number is rather small (241 of 2987 questions). Recently, we have researched ways to provide more useful feedback information to students. In [14] and [15] we have explored ways to use method of Formal Concept Analysis to give students meaningful visual representation (concept lattice digraph) of their performance on the course, and also quantitative measures (attribute coverage and formal concept similarity). In [16] we used model checking and mutation testing methods to propose a model for checking homework assignments consistency, and a model for generating richer feedback information for failed homework assignments.

In this research our motivation was to combine the approach of our homework calculation questions (dynamic inputs) with that of variable multiple choice questions (variable question text, answers and different images) with automated enriched feedback generation.

\section{Prototype Web application for automated question generation}

Most of the questions and assignments on our course Fundamentals of Electrical Engineering are based around a DC or AC circuit diagrams. We have also observed that in our e-learning system all of our circuit figures are static and had to be manually created, which is not preferable in an automated system. Furthermore, we know that only a small percentage of available questions in our system have rich (manually written) explanations. Therefore, we wanted to create a system which will automate question generation (including text and figures), as well as question solving and feedback information generation.

We decided to create a Web application for automated generation and solving of template based DC circuit questions. Therefore, our proposed Web application should meet following prerequisites:

- ability to generate simpler and more complex question variants form a basic question template: 
$\circ$ by changing the number of input parameters

o by increasing/decreasing complexity of the circuit

$\circ$ by changing the number of requested results

- automatic generation of random circuit figures:

○ series - parallel DC circuits

o one voltage or current source

- automatic solving of the given problem

- automatic generation of step-by-step solution to the given problem using basic circuit laws (Ohm's Law and the principles of Kirchhoff's Laws) as an additional feedback information for the students

\subsection{System overview}

Our Web application for automated generation of series - parallel DC circuit questions and solutions is available online at http://osnove.tel.fer.hr/questions/DCcircuits.asp. The application is bilingual - it is available in Croatian and English. Its main page is shown in the Fig. 1. Our automated application can create questions of different complexity based on the number of input parameters. It can generate a simple problem (e.g. one resistor connected directly on a voltage or a current source) requiring only one result (e.g. power dissipated on the resistor), and also very complex series - parallel circuits requiring 10 different results (total resistance, unknown resistances, voltage drop across resistors or the source terminals, current through resistors or the source and power dissipated by resistors or generated by the source). In its present setup students can choose between easy questions ( $1-4$ resistors), normal questions ( $5-9$ resistors) and hard questions ( $10-14$ resistors). Generating questions with more than 14 resistors is, of course, possible but the circuit image would not fit usual screen sizes. Every question has its own randomly generated circuit diagram figure. Also, during the automated solving process the circuit diagram will be randomly simplified step-by-step, by calculating equivalent resistances. The whole problem solving process is based on basic circuit laws - Ohm's Law and the principles of Kirchhoff's Laws.

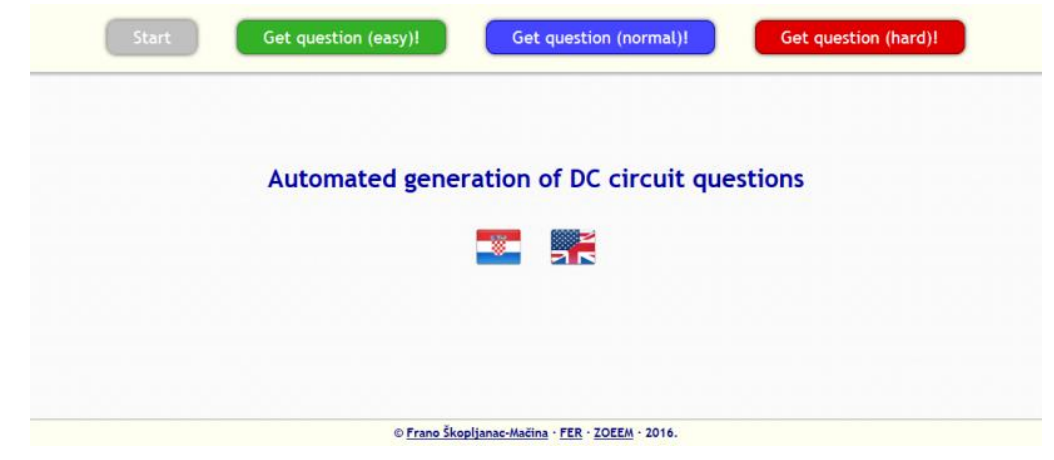

Fig. 1. Web application for automated question generation http://osnove.tel.fer.hr/questions/DCcircuits.asp

\subsection{Technologies}

We used standard Web technologies, such as HTML, JavaScript and jQuery [17]. Circuit diagrams are automatically drawn as a SVG [18] inline image. Mathematical notation is provided using JavaScript library MathJax [19] using standard LaTeX syntax. Microsoft ASP server-side scripting language is also used, but only for the Web application homepage. The core of our prototype application is client-based. Circuit diagram is drawn in SVG inline mode, which means that the image is not server-based, but generated directly on the client's browser from the generated SVG inline code. Still, we must acknowledge one downside of using SVG inline code - it does not work in Internet Explorer 8 and older Internet Explorer browsers.

\subsection{Question generation}

Question is generated by calling JavaScript function generateProblem (level). Therefore, first we need to decide on the difficulty level of the question (easy: up to 4 resistors; normal: form 5 to 9 resistors; hard: more than 9 resistors). Afterwards, the function randomly chooses the number of resistors (between defined lower and upper bounds), and then begins to form schematics (series - parallel circuit) as a nested brackets string of basic components $\mathbf{s}(* *)-$ two elements series and $\mathbf{p}(*, *)$ - two elements parallel. Schematic builder combines those two components by inserting (in place of a random star symbol) one into another and vice versa. It checks not to chain two same components one in another. Also it is possible to randomly extend number of elements between the brackets: $\mathbf{s}(*, *)$ can become $\mathbf{s}(*, *, *, *)$. Next, after the schematic string is complete the stars symbols are iteratively replaced by numbered resistors - e.g. $s(p(r 1, r 2), r 3, p(r 4, r 5))$. 
In the following step the functions decides randomly if this resistor combination will be connected to a voltage source or a current source. Afterwards, all the resistors are given a random value (between predetermined value ranges), as well as only one of the following: source voltage, source current or source power.

Next, the question is completed by deciding which of the unknown values need to be calculated, with random selection from the list of missing source parameters, and also from the list of missing resistors' voltages/currents/powers. Afterwards, the application generates the full text of the question. The text is created simply by appending the unknown values and their description to the simple template ("For the circuit shown in the figure, calculate..."), and by typing the list of known input data. Finally, the question is shown on the screen, together with the generated circuit diagram image (Fig. 2.). After solving the problem on paper students can check the results and stepby-step solution.

Currently, our system supports only this basic question type, but we plan to add different new question types by choosing different set of known values, e.g. by providing a current through one of the resistors, all resistor values, but no information about the voltage or current source parameters.

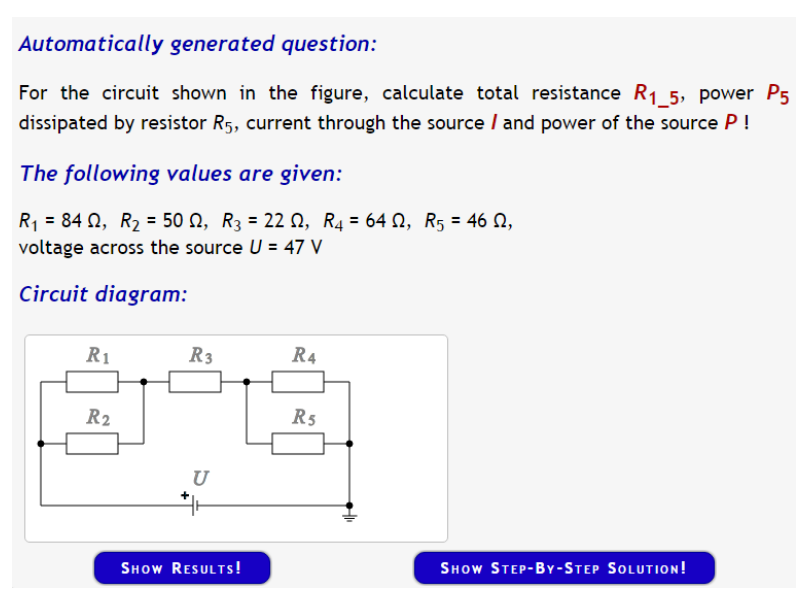

Fig. 2. Automatically generated question for schematic $\mathbf{s}(\mathbf{p}(\mathbf{r} \mathbf{1}, \mathbf{r 2}), \mathbf{r 3}, \mathbf{p}(\mathbf{r} 4, \mathbf{r 5}))$

\subsection{Circuit diagram drawing}

Web application converts schematic into a SVG inline image of a circuit diagram using prepared SVG symbols definition library shown on Fig. 3. Resistor, voltage source and current source are all 100 px wide and 20 px high so it is easier to combine them into a series - parallel circuit. Dot symbol is placed on all exact places where more than two lines meet. Ground symbol is always placed in the bottom right corner of the circuit diagram - on the sources negative terminal. Circuit diagram drawing functions are one of the more complicated parts of our application. Main function for circuit drawing is drawSchema(str). It is a recursive function which runs through the given schematic, by dividing it on smaller sub-schematics, and ensures that all resistors and line segments connect into their place.

After the series-parallel schematic is created it must be connected to the source. That is done in the final steps of the circuit drawing. Another function highlightedSchematics(find,type) can highlight in different colours various parts of the circuit diagram. This function is used extensively in feedback generation - to point out the set of resistors in series or in parallel which can be replaced by a single equivalent resistor.

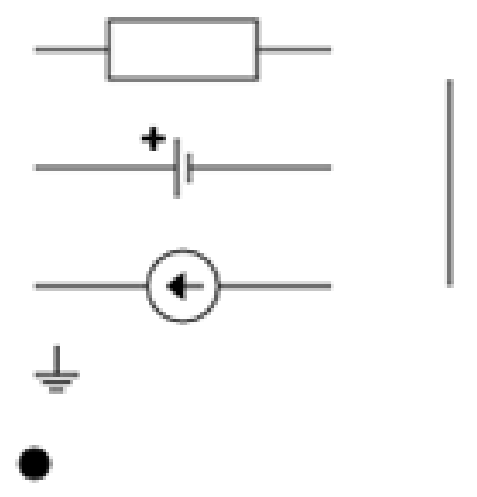

Fig. 3. Custom SVG definition library for DC circuits

\subsection{Problem solving and feedback generation}


After the question has been set up we can request numeric solutions to the problem by pressing button Show Results, or we can get entire step-by-step solution of the problem by pressing the button Show Step-By-Step Solution. We have defined a single strategy for solving questions in Section 3.4 (we know all the resistor values and one parameter of the voltage/current source). Function solveProblem(type) starts by iteratively simplifying starting circuit diagram, replacing random series or parallel of resistors with their equivalent resistances. After the circuit is reduced to one equivalent resistor, the function can calculate, using Ohm's Law and formulas for power, all the missing parameters of the source. After the total resistance is known, together with the source voltage, current and power the problem solving function moves in the opposite direction - replacing equivalent resistors with their predecessors. At the same time function calculates missing voltage, current and power of every resistor. Finally, the circuit diagram returns to its original state, and in that last step the functions calculates all the remaining unknowns.

Step-by-step solution provides a rich feedback for the students, with little hints, messages and specially highlighted circuit diagrams. Each step of the problem solving function is also typed out in detail using MathJax mathematical notation and calculated values.

When we add different types of questions, as hinted at the end of Section 3.4., we should also prepare different adapted strategies for problem solving. But, in essence they would also use only Ohm's Law and the main principles of Kirchhoff's Laws.

\section{Results and discussion}

Our method and resulting prototype Web application has shown promising results in automated generation of DC circuit questions. In Fig. 4 we can see another generated question with a circuit diagram containing 5 resistors. If we compare it to the question shown in Fig. 2, we can observe that we got two quite different questions of similar complexity. In both questions we got a voltage source, but the schema configuration is slightly different, and, besides total resistance, we need to calculate completely different unknown values. Of course, both questions have different results, and different step-by-step solutions. This illustrates that we can generate a list of different questions of the same complexity - which can be ideal for testing many students simultaneously. We could say that virtually all students in a normal sized group (80 students) would get unique - personalized questions of same difficulty. This approach also sounds promising in generating a large number of personalized homework assignments. But, in this prototype stage we will offer this application to our students mainly for self-assessment, practice and training for the written and oral exams.

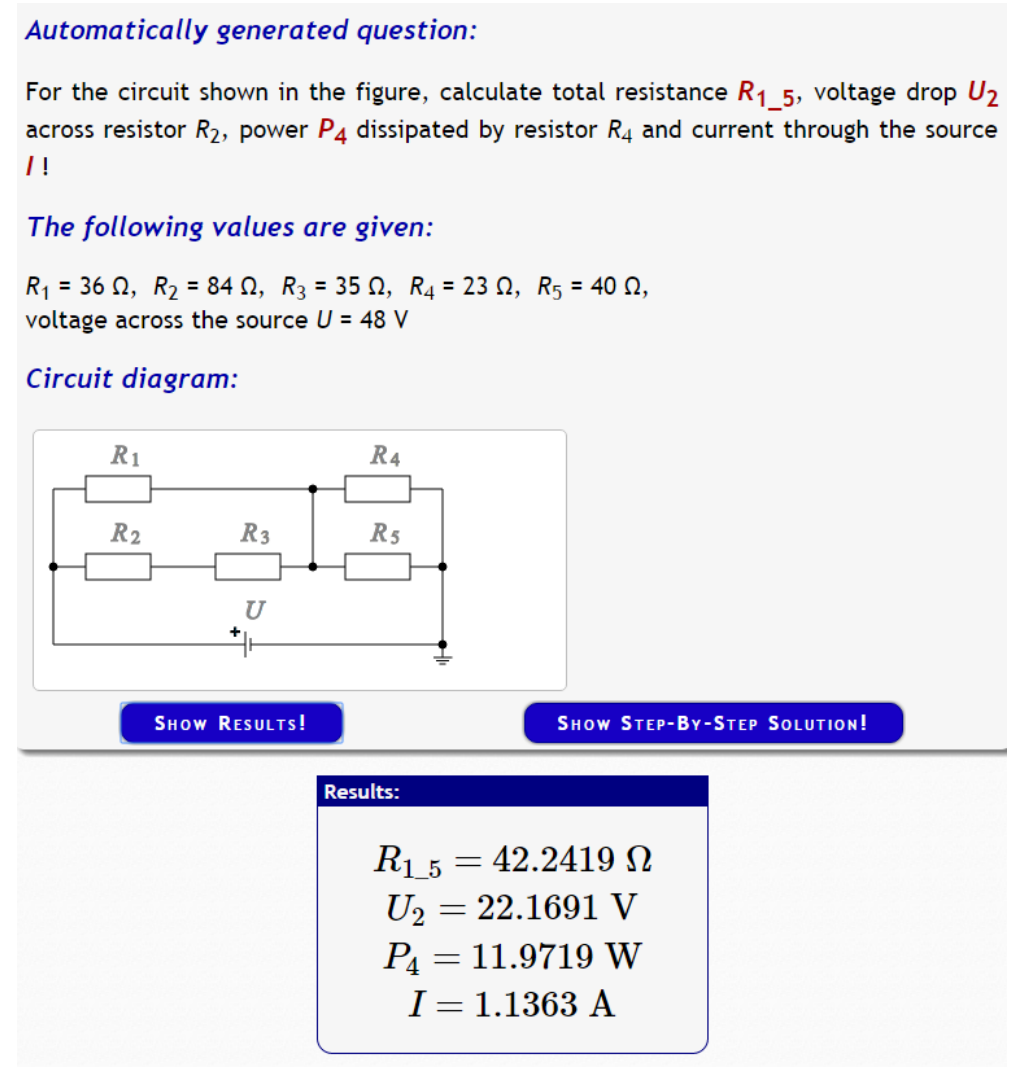

Fig. 4. Randomly generated question and its solutions (difficulty - normal)

Another example of our application's versatility can be seen in Fig. 5 where we have 4 different randomly generated DC circuit diagrams, each consisting of 7 resistors in different configurations. Of course, each circuit diagram is 
accompanied by a randomly generated text, with different combination of know values and requested unknown values. Also, for each resistor combination we can change the source type (voltage source to current source and vice versa). This increases the number of possible variations of questions with exactly 7 resistors. And by adding new question types, different sets of known and unknown data as pointed out in Section 3.4., the number of variations would further increase.

We can also see in Fig. 5 that all four different random circuit diagrams are not equally complex. Circuit diagrams in Fig. $5 \mathrm{c}$ ) and b) are less complex, as they require only one and two simplifications respectively (substitutions of parallel resistors with their equivalent resistor) to become a simple series resistor circuit. Circuit diagram in Fig. 5 a) is more complex, because it requires 3 simplification steps before it transforms in a simple parallel circuit (substitution of $R_{3}$ and $R_{4}$ resistor parallel with an equivalent resistor, and then substitute resistor series in both parallel branches with their equivalent resistors). Finally, circuit diagram in Fig. 5 d) appears to be most complex, because it needs 5 simplification steps to transform it into an equivalent simple parallel circuit. We start with the simplification of the $R_{3}$ and $R_{4}$ resistor parallel, then we substitute the resulting equivalent resistor $R_{34}$ and $R_{2}$ series with another equivalent resistor $R_{234}$, afterwards we calculate the $R_{234}$ and $R_{5}$ resistor parallel, and finally substitute resistor series in both parallel branches with their equivalent resistors $R_{12345}$ and $R_{67}$. Now, we have an equivalent simple parallel circuit of resistors $R_{12345}$ and $R_{67}$.

Therefore, it is necessary to describe these random circuit diagrams not only with the number of resistors, but also with an additional objective complexity measure. One example could be the number of nested parallel and series connections in the circuit diagram. This would correspond with the described number of simplification steps. Using that measure we could state that the circuit in Fig. 5 c) and Fig. 5 b) have circuit complexity 1 and 2 respectively. Circuit in Fig. 5 a) has circuit complexity 3, and the most complex circuit in Fig. 5 d) has circuit complexity 5. By applying this calculated circuit complexity measure the system could ensure that all student get different questions of the same circuit complexity. This measure is especially required in automatic student evaluation, because then we would avoid situations where one student gets a simple circuit diagram (Fig. 5 b)), and another gets a more complex circuit (Fig. 5 d)). The same measure could be also useful in self-assessment and tutoring, because the system could adapt to the current knowledge level of the student, by gradually increasing or decreasing circuit complexity.

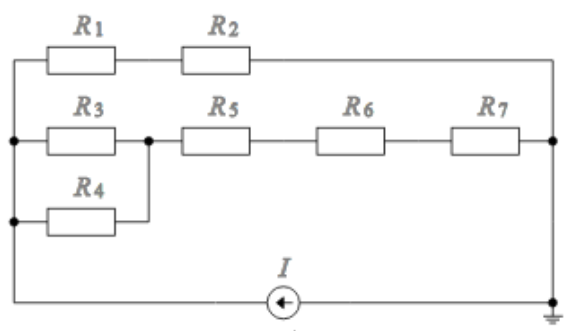

a)

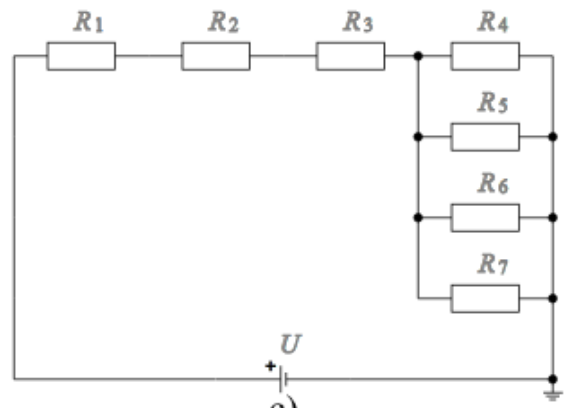

c)

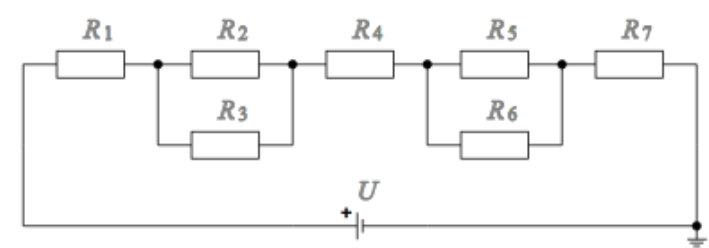

b)

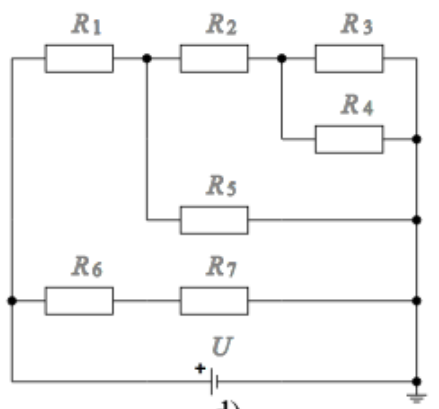

d)

Fig. 5. Four different random circuit diagrams with 7 resistors each

As we have described in Section 3.6., our Web application can calculate and display correct results, as shown in Fig. 4 , but also it can provide students with detailed feedback in form of step-by-step solution. The system displays all steps of the calculations using MathJax for mathematical notation, and additional explanations and hints. One example is presented in Fig. 6, where we can see a step 4 of a longer calculation process. In this step we identify simplification candidates, randomly choose one of the simple resistor parallels or series and calculate their equivalent resistance. By randomly simplifying the original circuit diagram the system also ensures that the same question can be solved in a number of different ways.

At this moment our web application works as a prototype, but it will show its full potential when we fully integrate it into our e-learning system. Also, we plan to add more question types, and different strategies for problem solving, namely using current divider rule and voltage divider rule. Additionally, it is possible to assign potentials to nodes in circuit diagrams, and that would further increase the number of question variations. In the next phase of development of our automated question generator we could include questions about Thévenin and Norton theorems and also maximum 
power theorem. Furthermore, the same principals described here could be applied to DC Capacitor networks and later, possibly even to AC circuits.

Before using our application in student evaluation (e.g. laboratory quizzes or homework assignments) we should conduct a proper verification and validation of our system using formal methods such as model checking. Also, we should test the outputs of our application by comparing them to results of a simulation of the same DC circuit in professional electronic circuit simulator (e.g. SPICE). Currently, the application was tested only manually by experts comparing system outputs to correct calculated results.

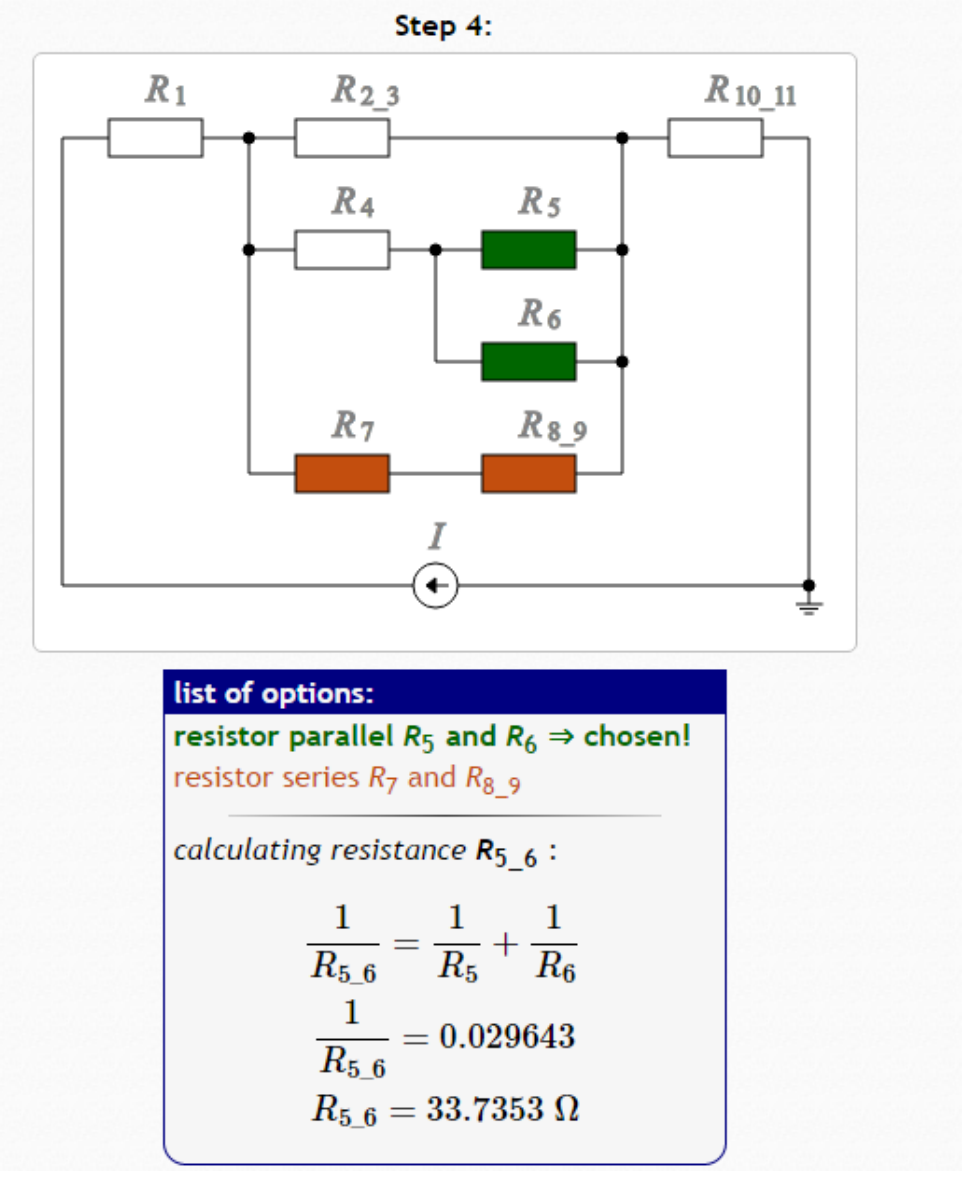

Fig. 6. Excerpt from step-by-step solution (difficulty - hard)

\section{Conclusion and future work}

In this paper we have presented our method and prototype Web application for automatically generating DC circuit questions. This method has the potential to help solve one of our main problems, and that is static nature of many teaching and learning materials. One of the main contributions of this paper is the circuit diagram random generator using only standard Web technologies. The other is the rich feedback generator offering step-by-step solution to randomly created DC circuit problem.

Currently, the presented Web application supports only one basic type of questions, and has one defined strategy for solving the generated problem. We plan to add other different types of questions and provide adaptive solving strategies.

In future work, we intend to improve our application prototype, making it more interactive so that the students can try different options in step-by-step solutions, and submit and check their answers automatically. Also, we intend to fully integrate presented application into our e-learning system, as a part of a developing adaptive module for student tutoring and knowledge evaluation.

\section{Acknowledgments}

The work has been fully supported by Croatian Science Foundation under the project (UIP-2014-09-2051 eduMINE Leveraging data mining methods and open technologies for enhancement of the e-learning infrastructure).

\section{References}


[1] Lin, X. \& Hu, Q. (2015). Trends in e-learning research from 2002-2013: A co-citation analysis, 15th IEEE International Conference on Advanced Learning Technologies, ICALT 2015, Hualien, Taiwan, July 6-9, 2015 , G. Sampson D.; Huang R.: Hwang G.; Liu T.; Chen N.; Kinshuk \& Tsai C. (Eds.), pp. 483-485, IEEE

[2] Grubisic, A.; Stankov, S. \& Zitko, B. (2015) Adaptive courseware: A literature review, J. UCS, vol. 21, no. 9, pp. 1168-1209

[3] https://moodle.org/, (2016). Moodle learning platform, Accessed on 2016-10-13

[4] Rakic, K.; Bandic Glavas, M. \& Majstorovic, V. (2013). Development of the Intelligent System for the use of University Information System, 24th DAAAM International Symposium on Intelligent Manufacturing and Automation, Zadar, Procedia Engineering 69 (2014) pp. 402-409

[5] Pryanichnikov, V.E.; Katalinic, B.; Kirilchenko, A.A.; Khelemendik, R.V.; Kuvshinov, S.V.; Vician, D. \& Uglesic, A. (2014). New Creative Educational Technologies for Inter-University Network, 25th DAAAM International Symposium on Intelligent Manufacturing and Automation, Vienna, Procedia Engineering 100 (2015) pp. 259-268

[6] Chali, Y \& Hasan, S. A. (2012). Towards Automatic Topical Question Generation, COLING 2012, Mumbai, Proceedings of COLING 2012: Technical Papers (2012) pp. 475-492

[7] Gulwani, S., (2014). Example-based learning in computer-aided STEM education, Commun. ACM, vol. 57, no. 8, pp. $70-80$

[8] Singh, R.; Gulwani, S. \& Solar-Lezama, A. (2013). Automated feedback generation for introductory programming assignments, in ACM SIGPLAN Conference on Programming Language Design and Implementation, PLDI '13, Seattle, WA, USA, June 16-19, 2013 Boehm H. \& Flanagan C. (Eds.), pp. 15-26, ACM, 2013.

[9] Alvin, C.; Gulwani, S.: Majumdar R. \& Mukhopadhyay S. (2014). Synthesis of geometry proof problems, Proceedings of the TwentyEighth AAAI Conference on Artificial Intelligence, July 27 -31, 2014, Québec City, Québec, Canada., Brodley, C. E. \& Stone P. (Eds.), pp. 245-252, AAAI Press

[10] Singh R.; Gulwani S. \& Rajamani S. K. (2012). Automatically generating algebra problems, Proceedings of the Twenty-Sixth AAAI Conference on Artificial Intelligence, July 22-26, 2012, Toronto, Ontario, Canada. Hoffmann, J. \& Selman, B. (Eds.), AAAI Press

[11] Zitko, B.; Stankov, S.; Rosic, M. \& Grubisic A. (2009). Dynamic test generation over ontology-based knowledge representation in authoring shell, Expert Syst. Appl., vol. 36, no. 4, pp. 8185-8196

[12] Pavić, A. \& Škopljanac-Mačina, F. (2012). Computer in testing the Fundamentals of electrical engineering at the University of Zagreb Faculty of electrical engineering and computing, MIPRO 2012 - 35th International Convention on Information and Communication Technology, Electronics and Microelectronics, Opatija

[13] Pardanjac, M.; Eleven, E. \& Kaurovic, D. (2014). Increase of User Motivation in Teaching Realized Through Distance Learning, Chapter 10 in DAAAM International Scientific Book 2014, pp.131-144, B. Katalinic (Ed.), Published by DAAAM International, ISBN 978-3-901509-98-8, ISSN 1726-9687, Vienna, Austria DOI: 10.2507/daaam.scibook.2014.10

[14] Škopljanac-Mačina, F.; Blašković, B. (2013). Formal Concept Analysis - Overview and Applications, 24th DAAAM International Symposium on Intelligent Manufacturing and Automation, Zadar, Procedia Engineering 69 (2014) pp. 1258-1267

[15] Škopljanac-Mačina, F.; Blašković, B. \& Skočir, Z. (2014). Using Formal Concept Analysis for student assessment, Proceedings of ELMAR-2014 56th International Symposium, Zadar, Tralić, D.; Muštra, M. \& Zovko-Cihlar, B. (Eds.), pp. 285-288 Zagreb, LotusGRAF, Zagreb

[16] Škopljanac-Mačina, F.; Zakarija, I.; Blašković, B. (2015). Exam questions consistency checking, Proceedings of 38th International Convention on Information and Communication Technology, Electronics and Microelectronics MIPRO 2015, Biljanović, P. (Ed.), pp. 921-924, GRAFIK, Rijeka

[17] https://jquery.com/, (2016). jQuery, Accessed on 2016-10-03

[18] https://www.w3.org/Graphics/SVG/ (2016). SVG, Accessed on 2016-10-03

[19] https://www.mathjax.org/ (2016). MathJax, Accessed on 2016-10-03 